\title{
Mechanical Ventilation Competencies of the Respiratory Therapist in 2015 and Beyond
}

\author{
Robert M Kacmarek PhD RRT FAARC
}

\author{
Introduction \\ Respiratory Care in 2015 and Beyond \\ The Mechanical Ventilator \\ Application of Mechanical Ventilation \\ Modes of Ventilation \\ Initiation and Adjustment of Mechanical Ventilation \\ Disease-Specific Management \\ Analysis of Ventilator Waveforms \\ Monitoring of Mechanical Ventilation and Airway Management \\ Pharmacologic Agents and Laboratory Data \\ Communication and Teamwork \\ Adjuncts and Nonconventional Approaches \\ Understanding the Evidence \\ Certifications \\ Being a Consultant \\ Evidence Supporting This Role \\ Summary
}

\begin{abstract}
The evolution of critical care and mechanical ventilation has been dramatic and rapid over the last 10 years and can be expected to continue at this pace into the future. As a result, the competencies of the respiratory therapist regarding mechanical ventilation in 2015 and beyond are expected to also markedly increase. Respiratory therapists are expected to be the experts on the mechanical ventilator and all aspects of the application of mechanical ventilation. They will be considered consultants on all aspect of ventilatory support. This requires an expanded education in a number of areas. To achieve these levels of competency, as recommended by the third " 2015 and Beyond" conference, the entry level education of the respiratory therapist of the future must be at the baccalaureate level. Key words: respiratory care; respiratory therapist; manpower; education competencies; protocols. [Respir Care 2013;58(6):1087-1092. (C) 2013 Daedalus Enterprises]
\end{abstract}

Dr Kacmarek is affiliated with the Department of Anesthesiology, Harvard Medical School, and the Department of Respiratory Care, Massachusetts General Hospital, Boston Massachusetts.

Dr Kacmarek presented a version of this paper at the 51st RESPIRATORY CARE Journal Conference, "Adult Mechanical Ventilation in Acute Care: Issues and Controversies," held September 7 and 8, 2012, in St Petersburg, Florida.

\footnotetext{
Dr Kacmarek has disclosed relationships with Covidien, Hamilton, Maquet, Kimberly-Clark, Newport, KCI, and Bayer.

Correspondence: Robert M Kacmarek PhD RRT FAARC, Respiratory Care, Warren 1225, Massachusetts General Hospital, 55 Fruit Street, Boston MA 01460. E-mail: rkacmarek@partners.org.
}

DOI: $10.4187 /$ respcare. 02546 


\section{Mechanical Ventilation Competencies of the Respiratory Therapist in 2015 and Beyond}

\section{Introduction}

The profession of respiratory therapy emerged in the 1940s and 1950s. At that time the competencies required of a respiratory therapist (RT) were very different from those required today. ${ }^{1}$ In fact, the title then used to refer to these individuals, oxygen technicians, reflects to a great extent the primary role of these clinicians. Most of the activities of these early RTs revolved around the delivery of oxygen therapy; moving oxygen cylinders, setting up oxygen tents and nasal oxygen "catheters." In addition, oxygen technicians administered aerosolized pharmacologic agents, performed pulmonary function tests, and performed blood gas analysis. Only on occasion were they involved in the provision of ventilatory support, but this was usually technical support. Over the years the role of the RT evolved in parallel with the development of medicine, in particular critical care. Most specifically, the maturing of the profession of respiratory care can be linked to the development of the mechanical ventilator and the application of mechanical ventilation. ${ }^{2}$ Today the competencies required of the RT in the area of mechanical ventilation far exceed those of the oxygen technician of the past, and the competencies expected of the RT will continue to expand as we approach $2015 .{ }^{3}$ In this paper the specific competencies required of the RT in 2015 and beyond will be addressed.

\section{Respiratory Care in 2015 and Beyond}

Over the last few years the American Association for Respiratory Care, through its "2015 and Beyond" task force, has addressed how the profession of respiratory care needs to evolve in order to meet the clinical demands that are being placed on the practicing RT. Though a series of conferences and subsequent reports this task force defined the changing role of the RT and the competencies needed by the RT.1,2,4 The outcomes of these conferences define an RT who is educated at the baccalaureate level, who is the expert in respiratory care in all care settings, is an individual who is capable of educating others on the provision of respiratory care, and is viewed by the medical community as a consultant. This individual, as a result, must be highly competent in a number of areas (Table 1). First of all, they must be experts on the mechanical ventilator and the indications and pathophysiologies that lead to the need for mechanical ventilation. They must also be able to interpret laboratory data that affect the care of the mechanically ventilated patient and the pharmacology used to manage these patients, regardless of route of administration. They must be competent in the application of adjuncts to mechanical ventilation: aerosolized pulmonary vasodilators, prone positioning, and non-conventional forms of ventilatory support. In addition, they require a
Table 1. Essential Areas Where Competencies Are Required in 2015 and Beyond

All technical aspects of the mechanical ventilator

Indications for and pathophysiology requiring mechanical ventilation

Independent application of mechanical ventilation

Pharmacology of critical care

Adjuncts to mechanical ventilation

Evidence supporting the application of mechanical ventilation

Guidelines, standard order sets, and protocols

Effective communication

Advanced certifications

Research methodology and statistical methods

Management of the airway

Bedside monitoring

working knowledge of extracorporeal life support techniques. They must be knowledgeable of the evidence supporting the application of mechanical ventilation and capable of developing and implementing guidelines and protocols for the application of mechanical ventilation. They must be able to effectively communicate with all levels of clinicians. Depending on their specific practice, they must be basic life support, advanced life support, pediatric advanced life support, or neonatal life support certified and be able to manage artificial airways and perform those bedside monitoring techniques required of mechanically ventilated patients.

\section{The Mechanical Ventilator}

The RT of 2015 and beyond must be a technical expert on every aspect of the mechanical ventilator. They should be able to discuss all of the technical nuances of the mechanical ventilator. They should be able to compare the capabilities of one ventilator to the other. They should be able to discuss in detail the mechanism of action of all of the modes and adjuncts that exist on the mechanical ventilator. They should be able to discuss the monitoring available on the ventilator and the trending of data. The RT of 2015 and beyond should be able to address any question related to the technical capabilities of the mechanical ventilator. They should be the individual whom all other professions seek out when questions in this area arise.

\section{Application of Mechanical Ventilation}

The RT should be the individual consulted regarding all questions on the application of mechanical ventilation (Table 2).

\section{Modes of Ventilation}

Mechanical ventilators are no longer simple devices with only few approaches to ventilatory support. Today venti- 


\section{Mechanical Ventilation Competencies of the Respiratory Therapist in 2015 and Beyond}

Table 2. Competency Areas During the Application of Mechanical Ventilation

Modes of mechanical ventilation

Disease specific management approaches

Effects of application on the cardiopulmonary system

Waveform analysis

Identification and correction of asynchrony

Provision of lung-protective ventilation

lators may have 10 to 15 unique modes of ventilation. ${ }^{2}$ The RT of 2015 and beyond should be capable of defining the operational differences between each of these modes. They should also be capable of defining situations where each mode of ventilation is indicated and be able to reference the literature supporting the application of each mode of ventilation. Many of these modes will be closedloop controllers of ventilatory support, each potentially approaching ventilatory support from a unique perspective. Understanding how modes such as Proportional Assist Ventilation, Neurally Adjusted Ventilatory Assist, and Intellivent differ, and their individual advantages and disadvantages will be essential competencies of the RT.5,6 Because of the increasing complexity of future mechanical ventilators, the RT will be expected to be the individual capable of addressing questions regarding modes during daily rounds.

\section{Initiation and Adjustment of Mechanical Ventilation}

Increasingly, ICUs are implementing standard order sets, guidelines, and protocols for the management of mechanical ventilation that empower the RT to select the mode of ventilation and determine how specifically mechanical ventilation is applied.7,8 This empowerment requires that the RT is knowledgeable of the pulmonary and cardiovascular implications of the particular approach and have the ability to rapidly indentify problems or lack of efficiency and make the appropriate adjustments. This independence in the application of ventilatory support requires the highest level of competency of the RT.

\section{Disease-Specific Management}

The independent management of ventilatory support by protocols, guidelines, or standard order sets requires that the RT be competent in managing patients presenting with varying pathophysiologies. Specifically, they should be able to adjust ventilatory support to match the unique requirements of patients who present with the ARDS, COPD, and asthma, as well as the routine postoperative and trauma patient or patients with pulmonary or surface burns and the patient overdosing on a pharmacologic agent. In order to
Table 3. Competency Areas Regarding Monitoring and Airway Care

Hemodynamic monitoring

Dead-space and shunt analysis

End-tidal $\mathrm{CO}_{2}$ and volumetric $\mathrm{CO}_{2}$ analysis

Esophageal manometry

Pressure/volume relationship analysis

Work-of-breathing analysis

Endotracheal intubation

Bronchoscopy assist

Tracheostomy tube selection

Tracheostomy care and tube replacement

Customization of tracheostomy tubes

Determination of compliance and airway resistance

Noninvasive cardiac output analysis, $\mathrm{O}_{2}$ consumption, and metabolic rate determination

do this they must have an in-depth understanding of the evidence that supports the application of mechanical ventilation in these specific settings.

\section{Analysis of Ventilator Waveforms}

Every ICU ventilator on the market displays waveforms of pressure, flow, and volume. The primary reason for these waveforms is to determine if the patient is ventilating in synchrony with the ventilator ${ }^{9,10}$ and if the application of ventilatory support is within the physiologic limits that will minimize the likelihood of developing ventilatorinduced lung injury. The RT has to be knowledgeable of the limits of ventilatory support and competent in identifying, via waveform analysis, patient-ventilator asynchrony, since asynchrony has been associated with patient outcome. ${ }^{11,12}$ In addition, the RT should be capable of modifying ventilatory support such that auto-PEEP is limited, flow asynchrony is minimized, and trigger and cycle asynchrony is eliminated, and to determine when mode asynchrony is present and which modes are appropriate alternatives.

\section{Monitoring of Mechanical Ventilation and Airway Management}

Since all mechanically ventilated patients have the potential of developing hemodynamic instability, the RT must have a detailed understanding of the expected response when mechanical ventilation is applied, and of the approaches used to maintain hemodynamic stability (Table 3 ). Monitoring of respiratory and ventilatory function of critically ill patients has expanded greatly in the last decade. Not only must the RT be competent in the analysis and interpretation of blood gases, but they should be able to analyze and interpret dead space and shunting as well as exhaled $\mathrm{CO}_{2}$. Competency in both end-tidal and volumet- 


\section{Mechanical Ventilation Competencies of the Respiratory Therapist in 2015 and Beyond}

ric $\mathrm{CO}_{2}$ analysis is a must. Bedside assessment of compliance and resistance is expected in all ventilated patients, but in addition the RT must be competent in esophageal manometry, and assessment of work of breathing, pressure volume curves, oxygen consumption, metabolic rate, and noninvasive techniques to assess cardiac output. The ability to perform endotracheal intubation, insert arterial lines, and provide bronchoscopy assist are key competencies of the RT. The RTs of 2015 and beyond must also be competent in the management of both endotracheal tubes and tracheostomies. They should be able to select tracheostomy tubes specific for a given patient and be able to replace and manage the variety of tubes that are commercially available, as well as determine when speaking valves are indicated and tracheostomy tubes can be removed.

\section{Pharmacologic Agents and Laboratory Data}

All critically ill patients receive a variety of pharmacologic agents. Since many of these agents directly or indirectly affect respiratory function, the RT must have an in-depth understanding of these interrelationships. They must understand the effect sedatives and narcotics have on ventilatory function and appreciate the impact that these drugs have on the development of delirium and outcome of critically ill patients.

RTs must be able to assess the need for the various categories of agents administered by aerosol and be competent in all of the new administration technology. Critical care RTs must also understand the results of various laboratory analyses and the impact of abnormal findings on respiratory and ventilatory function. RTs should also be able to perform basic interpretation of chest $\mathrm{x}$-rays.

\section{Communication and Teamwork}

The key to collaborative function of any clinician in critical care is effective communication and teamwork. The RT is a primary component in the management of critically ill patients. As such they need to actively communicate with all caregivers in this setting. The RT should be presenting the respiratory/ventilatory status of the patient during rounding. They should be an integral part of establishing the daily care plan for patients. The RT should be able to effectively argue for a specific approach to ventilatory support and always function as a patient advocate. Communication is an essential competency of the RT in the management of the mechanically ventilated patient.

Understanding what is expected of a team member and being able to function as a team leader are also key competencies of the RT in critical care. Today's critical care unit is run by a team, each member having a unique area of contribution to the function of the team. Decisions should normally be made only after each member of the team
Table 4. Competency Areas Regarding Adjuncts and

Nonconventional Approaches to Ventilatory Support

Aerosolized/inhaled pulmonary vasodilators

Prone positioning

High-frequency ventilation

Extracorporeal gas exchange

presents their specific perspective and the pro and cons of specific topics are discussed in detail. RTs are also expected to be team leaders regarding certain aspects of patient care, coordinating weaning of a difficult patient, management of a difficult airway, and selection of a new mode of ventilatory support for a specific patient. Competencies in the area of teamwork and collaboration are key to the optimal function of the RT in the management of mechanically ventilated patients.

\section{Adjuncts and Nonconventional Approaches}

Adjuncts to mechanical ventilation have had varying levels of success. However, all are still being actively researched because of the high mortality of subgroups of patients requiring ventilatory support (Table 4). As a result, the RT must be competent in the areas of aerosolized/ inhaled pharmacologic agents affecting the pulmonary vasculature, the use of prone positioning, and the various forms of high-frequency ventilation. Numerous drugs administered via the respiratory system affecting the pulmonary vasculature are now available. These drugs were first used in ARDS but now are increasingly used in patients with cardiac disease, especially during the postoperative period. ${ }^{13,14}$ The RT must be competent in the pros and cons of use of individual drugs as well as the various approaches to administering these drugs.

Prone positioning has been promoted in the management of ARDS and recent meta-analyses indicate that prone positioning affects outcome in very severe ARDS. ${ }^{15}$ Thus, the RT must be competent in the selection of patients potentially benefiting from this approach and the actual positioning of patients.

The use of high-frequency ventilation techniques for the management of severe respiratory failure is highly controversial. ${ }^{16-18}$ However, since this is a hotly debated approach to ventilatory care, the RT must be knowledgeable of the pros and cons associated with its application and the results of randomized controlled trials. They also should be competent in the technical/clinical application of highfrequency ventilation if used in their institution.

Extracorporeal life support (ECMO) is also a hotly debated approach to the management of the patient with ARDS, ${ }^{19,20}$ but ECMO is increasingly being used in patients with various forms of heart failure, patients awaiting 


\section{Mechanical Ventilation Competencies of the Respiratory Therapist in 2015 and Beyond}

lung transplantation, and patients with COPD. ${ }^{21}$ The RT should be knowledgeable of the potential indications and contraindications for ECMO and, if utilized in their institution, competent in the application and management of the patient receiving ECMO.

\section{Understanding the Evidence}

In order for the RT to function in the role described, they must be capable of understanding and critiquing the increasing volume of evidence published regarding mechanical ventilation. In order to do this they must have a general understanding of research techniques and statistical methodology. Ongoing clinical forums discussing new literature should be available for all therapists. During rounds the RT should be able to discuss the nuances of newly published literature regarding mechanical ventilation.

\section{Certifications}

Certification is available at many levels, and increasingly clinicians are being expected to demonstrate their competency by becoming certified. Specifically, all RTs who work in an ICU should be both basic life support and advanced life support certified. If working in a neonatal or pediatric ICU, neonatal life support and/or pediatric life support certification is a must. In addition, the RTs in 2015 and beyond will be expected to demonstrate their competencies in critical care by being certified as neonatal/pediatric or adult critical care specialists by the National Board for Respiratory Care.

\section{Being a Consultant}

The expectation of RTs providing ventilatory support in 2015 and beyond is that they will function as consultants. They will be considered the experts in mechanical ventilation and should be regularly asked their opinion on how to manage the ventilatory aspects of patient care. Essentially, the expectation will be that if no one is interested in your opinion, you will be considered unnecessary. The RT should be considered by all as the expert in mechanical ventilation and regularly asked at rounds and team meetings to state their opinion on how care should be provided.

\section{Evidence Supporting This Role}

As part of the third 2015 and Beyond conference, questionnaires were sent to directors of respiratory therapy educational programs ${ }^{22}$ and directors of departments of respiratory therapy. ${ }^{23}$ The results of these surveys support the described role of the RT in mechanical ventilation. However, not all programs provide education in all of the areas described or at a level to ensure competency. The time available in the typical associate degree program is simply insufficient to ensure that all graduates have obtained these competencies. It is because of the expectations of the medical community and the limited time for education at the associate degree level that the 2015 and Beyond task force recommended that all educational programs in respiratory care be transitioned to baccalaureate degree granting programs by the year 2020 .

A very disappointing aspect of the entire 2015 and Beyond process was the response of the directors of respiratory therapy departments to the surveys. Surveys were sent on multiple occasions to the American Association for Respiratory Care members list of directors and managers of respiratory therapy departments, and the response rate from this group was $28 \%$, compared to a response rate of $80 \%$ from directors of respiratory therapy educational programs.

\section{Summary}

The role of the RT and, as a result, the competencies of the RT in all aspects of patient care, but especially mechanical ventilation, is expected to expand in 2015 and beyond. This expanded role can be best defined as an expert consultant in the provision of mechanical ventilation. In order for the profession to be able to train the new generation of RTs for this role, the educational programs in respiratory care need to expand to the baccalaureate level, as recommended by the third 2015 and Beyond conference. $^{4}$

\section{REFERENCES}

1. Kacmarek RM, Durbin CG Jr, Barnes TA, Kageler WV, Walton JR, O'Neil EH. Creating a vision for respiratory care in 2015 and beyond. Respir Care 2009;54(3):375-389.

2. Kacmarek RM. The mechanical ventilator: past, present, and future. Respir Care 2011;56(8):1170-1180.

3. Barnes TA, Gale DD, Kacmarek RM, Kageler WV. Competencies needed by graduate RTs in 2015 and beyond. Respir Care 2010; 55(5):601-616.

4. Barnes TA, Kacmarek RM, Kageler WV, Morris MJ, Durbin CG Jr. Transitioning the respiratory therapy workforce for 2015 and beyond. Respir Care 2011;56(5):681-690.

5. Kacmarek RM. Proportional assist ventilation and neurally-adjusted ventilatory assist. Respir Care 2011;56(1):140-148.

6. Sulemanji D, Marchese A, Wysocki M, Kacmarek RM. Adaptive support ventilation with and without end-tidal $\mathrm{CO}_{2}$ closed loop control vs conventional ventilation. Intensive Care Medicine 2013;39(4):703-710.

7. Haas CF, Loik PS. Ventilator discontinuation protocols. Respir Care 2012;57(10):1649-1662.

8. Ellis SM, Dainty KN, Munro G, Scales DC. The use of mechanical ventilation protocols in the intensive care unit: a survey of current practice. Crit Care 2012;27(6):556-563.

9. Murias G, Villagra A, Blanch L. Patient ventilator synchrony during assisted invasive mechanical ventilation. Minerva Anestesiol 2013; 79(4):434-444.

10. Di Marco F, Centanni S, Bellone A, Messinesi G, Pesci A, Scala R, 


\section{Mechanical Ventilation Competencies of the Respiratory Therapist in 2015 and Beyond}

et al. Optimization of ventilator setting by flow and pressure waveforms analysis during noninvasive ventilation for acute exacerbations of COPD: a multicentric randomized controlled trial. Crit Care 2011;15(6):R283-R288.

11. Thille AW, Rodriquez P, Cabello B, Lellouche F, Brochard L. Patient ventilator synchrony during assisted mechanical ventilation. Intensive Care Med 2006;32(10):1515-1521.

12. de Wit M, Miller KB, Green DA, Ostman HE, Gennings C, Epstein SK. Ineffective triggering predicts increased duration of mechanical ventilation. Crit Care Med 2009;37(10):2740-2745.

13. Khan TA, Schnickel G, Ross D, Bastani S, Laks H, Esmailian F, et al. A prospective, randomized, crossover pilot study of inhaled nitric oxide versus inhaled prostacyclin in heart transplant and lung transplant. J Thorac Cardiovasc Surg 2009;138(6):1417-1424.

14. Antoniou T, Prokakis C, Athanasopoulos G, Thanopoulos A, Rellia $\mathrm{P}$, Zarkalis D, et al. Inhaled nitric oxide plus iloprost in the setting of post-left assist device right heart dysfunction. Ann Thorac Surg 2012; 94(3):792-798.

15. Sud S, Friedrich JO, Taccone P, Polli F, Adhikari NK, Latini R, et al. Prone ventilation reduces mortality in patients with acute respiratory failure and severe hypoxemia: systematic review and metaanalysis. Intensive Care Med 2010;36(4):585-599.

16. Derdak S, Mehta S, Stewart TE, Smith T, Rogers M, Buchman TG, et al; Multicenter Oscillatory Ventilation For Acute Respiratory Distress Syndrome Trial (MOAT) Study Investigators. High-frequency oscillatory ventilation for acute respiratory distress syndrome in adults: a randomized, controlled trial. Am J Respir Crit Care Med 2002;166(6):801-808
17. Ferguson ND, Cook DJ, Guyatt GH, Mehta S, Hand L, Austin P, et al; OSCILLATE Trial Investigators; Canadian Critical Care Trials Group. High frequency oscillation in early acute respiratory distress syndrome. N Engl J Med 2013;368(9):795-805.

18. Young D, Lamb SE, Shah S, MacKenzie I, Tunnicliffe W, Lall R, et al; OSCAR Study Group. High frequency oscillation for the acute respiratory distress syndrome. N Engl J Med 2013;368(9): 806-813.

19. Tài Pham T, Combes A, Rozé H, Chevret S, Mercat A, Roch A, et al; for the REVA Research Network. Extracorporeal membrane oxygenation for pandemic influenza A (H1N1): induced respiratory distress syndrome: a cohort study and prospective matched analysis. Am J Respir Crit Care Med 2013;187(3):276-285.

20. Peek GJ, Mugford M, Tiruvoipati R, Wilson A, Allen E, Thalanany $\mathrm{MM}$, et al; for the CESAR trial collaboration. Efficacy and economic assessment of conventional ventilatory support versus extracorporeal membrane oxygenation for severe adult respiratory failure (CESAR): a multicentre randomised controlled trial. Lancet 2009;374(6):13511363.

21. Diaz-Guzman E, Hoopes CW, Zwischenberger JB. The evolution of extracorporeal life support as a bridge to transplantation. ASAIO J 2013;59(1):3-10.

22. Barnes TA, Kacmarek RM, Durbin CG Jr. Survey of respiratory therapy education program directors in the United States. Respir Care 2011;56(12):1906-1915.

23. Kacmarek RM, Barnes TA, Durbin CG Jr. Survey of directors of respiratory therapy departments regarding the future education and credentialing of respiratory care students and staff. Respir Care 2012; 57(5):710-720

\section{Discussion}

Branson: I've worked in the department of surgery since 1985 . I don't work in the respiratory therapy department, and I don't supervise respiratory therapists [RTs] at the bedside, but I like everything you said. At present, where are we falling down? Is it the abilities of students and getting good applicants to the schools? Is it at the schools? Is it at the hospitals? Where is the next generation of RTs who will lead the science and the profession coming from?

Kacmarek: I don't know if that's true. You know, the older we get, the more we think we know and the less we think new graduates know. The reality is that the new graduate today knows a lot more than we did when we entered the profession. And 20 or
30 years ago, when a similar group sat here, they probably said the same thing: "There's nobody coming after us to take our place, and where are all the young people?" There are people out there, but I don't think there are enough of them. I don't think we always attract the right individuals into respiratory care, and that's partly because of the entry-level academic credential of the profession.

When you talk to your children about their future profession, is respiratory therapy ever a part of the discussion? When I talk to my children, my expectation is that they will get at least a baccalaureate degree. With a profession that has as its entry level an associate degree, it eliminates a lot of students whose goal is a profession that requires a baccalaureate degree. You don't see school guidance counselors even talking about respiratory care, because it does not require a baccalaureate at the entry level.

I believe we have problems at the respiratory therapy department level, and I'll say it, since I'm one of that group, many respiratory therapy department directors are not as interested in improving the quality of care as they are in maintaining the fiscal viability of their departments. They're not focused on quality; they're only focused on financial issues. Now, obviously, that's a generalization that doesn't fit across the board, but only $27 \%$ of the directors of respiratory therapy programs provided input into a very important survey that will help determine the future of respiratory care. What happened to the other $73 \%$ who chose not to even participate in that process?

I think we have problems at multiple levels. I think we have problems 


\section{Mechanical Ventilation Competencies of the Respiratory Therapist in 2015 and Beyond}

with the educational entry level that eliminates good people, and we clearly have problems in our current leadership at the hospital level, and that needs to be improved.

Branson: Chris Blakeman has worked with me now in the department of surgery for the past 5 years, but up until Chris, all the people I worked with closely-and I would consider some people I mentored-all of them either work for industry, went into medical school, or became PAs [physician assistants], perfusionists, or CRNAs [certified registered nurse anesthetists]. That's part of what the educators seem to be fighting: how important it is to have a bachelor's degree and create an advanced degree, because we lose a lot of the best people due to just financial reasons. There are just so many academic jobs that provide a reasonable living, and I think that's really important and gets lost. We lose so many people from the profession because the only upward career pathway is into administration. There are no advanced practice RTs.

Kacmarek: One of the problems with salaries is those we are compared to! I'm frequently sitting next to physical therapists with $\mathrm{PhDs}$ at entry level, occupational therapists with master's degrees at entry level, or speech/language pathologists with master's degrees or PhDs at entry level. All those people have a much higher academic entry point than RTs. As a result, we're at the end of the line.

MacIntyre: Let me switch from the entry level to the top. I think that the profession as it's constructed right now has a ceiling above which you can't go without leaving the profession and doing something else. But at Duke we've built a huge new bed tower with a bunch of new ICU beds and we're running around like chickens with our heads cut off trying to figure out how to staff it.
An obvious staffing model would be a PA-RRT. I can think of no better entry level for a PA than the RRT to move into an ICU and be an independent practitioner. It seems to me the next step for the respiratory care profession is to create independent practitioner licensure by the states, just like the physical therapists, occupational therapists, PAs, and CRNAs to provide independent care. Rather than count somebody who goes to school for PA as lost to the profession, instead somehow make it possible that they're still within the profession. I know there are a bunch of RTs who would love to see independent licensure for advanced level RTs.

I think it's a terrific idea, but it's going to be a huge uphill battle to get state legislatures and all the things that need to go into place to get licensure for independent practice. Why not somehow partner with the PA system that's already in place, already grants a master's degree, and in all 50 states can get a license? Rather than consider them lost RTs, consider them RTs who have broken through the ceiling and can provide independent expertise. In my ICUs I have about $10 \mathrm{RTs}$ who'd be terrific if I could get them to be independent practitioners.

\section{Kacmarek: You could retire!}

MacIntyre: With a PA licensure they could damn near run my ICUs.

Kacmarek: The problem is that too few individuals are entering the profession with the capability of moving in the direction Rich described, because our entry-level requirement immediately makes them choose another profession.

MacIntyre: Oh, I realize it will be few.

Kacmarek: Because of the current entry-level criteria. One of the arguments against requiring a baccalaureate degree is that we'll have nobody applying to respiratory therapy programs. I disagree with that entirely; a baccalaureate entry opens up a broad spectrum of high school graduates who would never have considered respiratory care because of its associate's degree entry, as opposed to a baccalaureate. Today we have 54 baccalaureate level respiratory care programs and 3 quasi-master's respiratory care programs. They're master's programs that provide a degree, but they're not clinically oriented.

MacIntyre: I'm not arguing with you on the entry-level part of it. It just seems to me that the respiratory care profession would have more attraction if our superstars had an avenue to proceed through that ceiling.

Kacmarek: No question. We can even look at the fact that if we had baccalaureate entry, why couldn't we have advanced level RTs with master's degrees, like nurses do? They're your PAs by virtue of their educational level. It opens up the opportunity for upward mobility to a much greater extent. I agree with Rich that, unless you move into education or management, the profession doesn't have a mechanism for upward advancement.

MacIntyre: One last pitch for the American Respiratory Care Foundation here. One of the topics on their agenda is focusing the scholarship program away from associate level scholarships and consolidating them into really meaningful advanced degree scholarships. I think the advanced degree scholarships should have licensure attached, to allow breaking through that ceiling and into a clinical leadership role.

Kacmarek: Agreed.

Kallet: I would like to offer a counter viewpoint or a cautionary viewpoint on this. These discussions are extremely important, and I have a tremendous amount of sympathy for 


\section{Mechanical Ventilation Competencies of the Respiratory Therapist in 2015 and Beyond}

what's been said. However, in the context of a shrinking middle class, I think back to my own roots. I entered respiratory therapy as a teenager in Syracuse, New York, during the deep economic recession of the 1970 s, when my dad was unemployed. An associate's degree was something doable and attainable. The model at Upstate Medical Center was extremely good: it was a "two-plus-two" program. You got in, you got great associate's degree level training, and then the upper division course work was advanced respiratory care. There was the option of supporting yourself while working towards a bachelor's degree.

Parenthetically, in northern California, the RT graduates we've hired from our community college programs have been great. They're bright, well prepared, and a joy to work with. They're ready for us to train them into the much more advanced role we need them to take on. But a lot of them do not have the economic means to go through 4 years of college in one bite. They're not from upper middle class or solid middle class families. They're kids, a lot of them from immigrant families, working to get into the middle class.

While I totally understand where the profession needs to go, and if we try we can get the profession to where it needs to be, but to move it to a baccalaureate program where all you're doing is saying the kids need 2 years of basic college education, then they get the associate's program core courses in the last 2 years ...

Kacmarek: You said that: I didn't say that.

Kallet: OK, but in a lot of the baccalaureate programs I'm aware of it's not really advanced respiratory care. The training I went through when I went back and got a baccalaureate was advanced respiratory care, advanced biology and chemistry-subjects that made me a better professional: education, management, and research. If it goes that way, fine, but I think if the idea is to get this up to a level where all we're really doing is saying, "Go to a community college for 2 years and do the basic science and liberal arts, and then do 2 years of core respiratory care curriculum that's basically an associate's level degree," we're going to limit a lot of very bright young people who could enter the middle class and the profession without appreciably advancing their knowledge base.

Kacmarek: We are going to change the demographics of who enters the profession, there's no question. But the creativity associated with developing a baccalaureate program is unlimited. Nobody is proposing a restriction that will make it all private 4-year colleges that offer baccalaureate programs at \$50,000 a year. Many states will allow associate-degree-granting community colleges to develop a baccalaureate degree.

Florida has several schools that are in the process of moving from associate programs to baccalaureate programs. You can structure it any way you want. But we need to "bite the bullet" and accept that by some date we must start requiring baccalaureate degrees. And that at some later date all the associate degree programs have to become baccalaureate programs. Nobody is saying this is going to be done overnight; it has to be a reasonable transition. I have sympathy for what you're saying, but I also agree with Rich Branson that we need a better quality individual who is motivated to the levels he referred to; otherwise, the profession will not grow and develop: it will eventually die.

Kallet: I agree with that. I think it's a matter of how it's done. And I think the idea of still allowing people to come into respiratory therapy with an associate's degree, but with a contract expectation that, once they can earn money, they will continue their education and get a bachelor's degree ...
Kacmarek: There has to be a point in time where we have to "bite the bullet." We have to grow as a profession. Eventually, we have to say it has to change and everybody entering respiratory care has to be educated at a higher level.

Turner: Another issue is the importance of collaborative interdisciplinary education. I think that more and more we're moving towards trying to get all trainees to learn together earlier in their training. As an example, we have our pediatric critical care fellows shadow the RTs and the nurses early in their fellowships, to foster an interdisciplinary approach to their education. With improved collaboration, both education and patient care are enhanced, which is particularly important in the ICU environment, where teamwork is crucial.

Kacmarek: I agree. I think the ICU does it better than any other aspect of in-patient care, because of the nature of the work and the type of professionals who are there. There's a more collaborative attitude in most ICUs, so we can be the model for collaborative education in other parts of the institution.

Branson: I understand that the real issue is that we need to develop these educational constructs for people to grow within the system. Is there any advantage in funding internships? An internship at Massachusetts General Hospital for 6 months or a year? An internship at San Francisco General for a year? The question is, what kind of piece of paper do you get when you're done? Ideally, you'd get this wealth of experience and we'd be pushing these people out of the nest to hopefully fly to other places and create similar programs at other hospitals.

Kacmarek: You could do that. But it takes a long time for somebody to get acclimated to an insane asylum 


\section{Mechanical Ventilation Competencies of the Respiratory Therapist in 2015 and Beyond}

like our place. By the time you're acclimated, you're half way through the internship. I would be more in favor of funding other institutions who have the capability of moving to the level that we, and Duke, and the Mayo Clinic, and other places are, to assist them so that their departments become what our departments are.

Marini: Bob, do you agree with this historical perspective? One of the big reasons people aren't considering respiratory care is not only that energy has been sapped out of it by the business interests of the hospitals, but also that the doctors are no longer at the bedside. The mentors I learned from, Hudson and Pierson, were excited about physiology, which is no longer very well taught to our medical students.

The physiological intrigue of being an RT seems to have dissipated some, too. A lot of our RTs seem to have limited interest or encounter trouble with the waveforms, the interpretations, the reasons behind why the patient fails. They'll have an opinion as to why a patient failed a weaning trial or need support, but the physiological understanding has eroded because the medical profession has allowed physiology to take a back seat to statistics and evidence-based medicine. And doctors are more interested in getting on with their next patient than in mentoring someone who is not really well prepared to receive the information.

The whole field of medicine has changed radically, and not always for the better, in my opinion. You still have to use physiology to extricate the information and unravel the knot that most patients present. I think we have a great and dedicated group of RTs at my hospital, but even our RTs are often too busy applying treatments and documenting observations to ponder what might be happening underneath it all, and their high workload makes them eager to move on to the next patient. They cannot be with us on rounds much of the time. We try to educate them, but the culture has changed.

Kacmarek: A certain culture in the respiratory therapy department has to be promoted to allow that to happen. If I went into any ICU and asked the RTs if they are on rounds $100 \%$ of the time, the answer would be no, because they may have 6 or 7 patients, and this patient's tube needs to be re-taped, this is happening here, and that's happening there, so they get pulled away from rounds. They're not $100 \%$ on rounds because it's impossible, because of critical problems. But I agree that physiology is not taught to the level it should be. And the kids coming out of respiratory care schools do not understand physiology to the level they should.

But it's also important to remember the difference in our expectations of an associate degree graduate 20 years ago, compared to today. We expect that person to know a lot more than we did 20 years ago, but we haven't expanded the time and opportunity for them to learn it, so I can't expect them to come as prepared as I would like. We spend 3 months with them. Dean and I do one-on-one lecturing; Dean does workshops with them on how to start NIV. We do a lot of one-on-one education to train them to be the RTs we want at the bedside.

Branson: I agree with both of you. I grow tired of bedside caregivers telling me they made a change because they felt like the patient would do better-with no physiologic evidence or basis in the literature. I hear things such as, "I felt like ATC [automatic tube compensation] was a good idea on this patient during the spontaneous breathing trial, because he has a smaller tube." I don't know where the problems reside, and so I don't know how to fix them. I think this is a great opportunity for the profession, but I'm not sure how we move forward.
Giordano: $\quad$ I agree with everything that's been said, but eventually we have to put our feet on the ground and not be floating 30 feet in the air. I offer a couple things to think about. First, I think there's an inevitable move toward requiring RTs to get at least a baccalaureate degree. We already have over $50 \%$ of our members with at least a bachelor's degree. Could this be because they found that with an associate's degree their education was wanting?

I think a critical event that will shift this paradigm is the bill ${ }^{1}$ we're pushing to get RRTs who have baccalaureate degrees to work under Medicare Part B, as employees of physician practices. They will not bill independently; they'll be salaried by the practice. But they'll be able to see patients with chronic lung diseases and provide disease management in the physician's office without having to make an appointment with the physician. That will push even more people to acquire those grounding communication skills. It will happen, and it will drive change.

Regarding capacity, the RT profession has a burn rate of approximately 8,000 people per year, so we have to graduate that many per year. As you mentioned, we have approximately 55 baccalaureate degree programs and 3 graduate programs, but they're not filling up their classes. Another survey we conducted as part of the 2015 and Beyond initiative was to ask allied health teams whether they have what they need to develop respiratory care baccalaureate programs. We had only one positive response. That survey will be repeated with a predicate of this baccalaureate requirement to work in offices, and I think that will change then.

What it comes down to is the market. Bob mentioned this in regards to Florida, which I believe a couple of years ago changed its laws to allow

\footnotetext{
* Sam P Giordano MBA RRT FAARC, Publisher, Respiratory CARE, Irving, Texas.
} 
community colleges to offer baccalaureate degrees. This was done not because the educators and professions got together: it was because, across all occupations and professions, the employers said to the education system that the graduates are not adequately educated and you need to step it up. Everything is market-driven. Until every department head has a baccalaureate degree as a prerequisite, you will not necessarily have everybody with a baccalaureate degree.

Case in point: nursing has been advocating for baccalaureate-trained nurses for at least 3 decades, but over $50 \%$ of nurses in the workforce are associate-degree prepared, because that's what the marketplace accepts. When we change the marketplaceand many of the things that were discussed at this conference will feed into that - we will see this change, this evolution. We'll then be able to show that it's a sound business model for educators and universities to create baccalaureate programs.

One final point: a bit of a caution is indicated from the experience of our friends the physical therapists. A funny thing happened on the way to doctoral degrees, which is that all of a sudden there developed a physical therapy assistant, and guess where they're getting educated? At community colleges, and getting associate's degrees. So, whereas once there was, especially in hospitals, a team of, say, 20 physical therapists, now there may be 5 physical therapists and 15 physical therapist assistants. I don't know whether this will boomerang on us in a similar way, just because of the higher critical thinking that's going to be demanded in the future, but I would not necessarily hold that up as a road we want to go down. Certainly it's a design we've learned from.

Kacmarek: We're not asking for $\mathrm{PhD}$ entry, we're asking for baccalaureate entry. And if you allow the market to drive itself, we will all be dead before any change is made. It seems to me that we can do what's necessary to make change occur without damaging the workforce or entry into the workforce. Nobody has asked for this to happen tomorrow; what we are asking for is to start setting solid deadlines for things to change.

In Massachusetts we're working with 2 community colleges, and we believe we'll be able to go to baccalaureate degrees in community colleges in Massachusetts, like they have in Florida. Increasingly, I think we'll see that same thing happening in multiple states, because, as you said, it seems to be the right thing to do to properly educate people for various professions. Why can't we say that,
10 years from now, you can't develop a new respiratory therapy program unless it's at the baccalaureate level, and 20 years from now that all associate degree programs have to change to baccalaureate? That's easily enough time for the profession to acclimate to the changes that are being demanded of us to survive.

Giordano: I agree, but we have to make sure there's enough education capacity out there. We've got some university deans to talk to.

Kacmarek: Sure, but I also think that we make a bigger deal about the manpower issues than is real. In the northeast we have many RTs who don't have jobs. This year's graduates from respiratory therapy programs in Massachusetts, Rhode Island, Vermont, New Hampshire, Connecticut, and Maine can't find jobs. I've had 50-70 applicants for just a couple openings. We have lists of people who cannot find work in respiratory care in the northeast. I realize there are pockets throughout the United States where there are deficiencies, but I'll bet that, globally, recently that's changed a lot and that we have an excess of RTs.

\section{REFERENCE}

1. Medicare Respiratory Therapy Initiative Act of 2011. H.R.941. 112th Congress, first session. 